Madalina Chitez, Joachim Hoefele, Liana Konstantinidou

\title{
4 Korpora in der beruflichen Bildung: Grundlage einer bedürfnisorientierten Förderung der Schreibkompetenz
}

\begin{abstract}
Die Förderung der sprachlich-kommunikativen Kompetenzen im allgemeinbildenden Unterricht (ABU) an Berufsfachschulen erfordert - aufgrund der verschiedenen Sprachbiographien und heterogenen sprachlichen Kompetenzen der Lernenden - nicht nur eine systematische Erfassung der demographischen, migrations- und sprachbiographischen Merkmale der Lernenden, sondern auch eine differenzierte Analyse ihrer sprachlichen Fähigkeiten. Der hier vorliegende Beitrag zeigt, wie anhand eines Korpus von Lernenden-Texten mit korpuslinguistischen Methoden die sprachlichen Merkmale von Lernendentexten analysiert und beschrieben werden. Texte mit ähnlichen Merkmalen werden in Verbindung mit den demographischen, migrations- und sprachbezogenen Variablen gebracht. Dadurch können die sprachlich-kommunikativen Fähigkeiten der Lernenden erfasst und der Förderbedarf im Bereich (schrift)sprachlich-kommunikativer Kompetenzen gezielt ermittelt werden. Die Ergebnisse sollen eine empirisch gesicherte Grundlage zur Förderung der Grundkompetenzen bilden, hier insbesondere des Schreibens in der Berufsbildung, die der sprachlichen Heterogenität der Lernenden mit und ohne linguistischen Migrationshintergrund gerecht wird.
\end{abstract}

\section{Ausgangslage}

Die Förderung der sprachlich-kommunikativen Kompetenzen im allgemeinbildenden Unterricht (ABU) an Berufsfachschulen stellt aufgrund der verschiedenen Sprachbiographien und heterogenen sprachlichen Kompetenzen der Lernenden eine große Herausforderung dar. Studien zeigen, dass Berufsfachschüler/innen größtenteils Mängel im Lesen und Schreiben aufweisen (vgl. Efing, 2008; economiesuisse, 2010; Baumann, 2014).

Vor diesem Hintergrund stellt sich die Frage, wie die sprachlich-kommunikativen Kompetenzen der Lernenden in Hinblick auf die Heterogenität ihrer Sprachbiographien und die große Varianz ihrer sprachlichen Fähigkeiten am besten zu fördern sind (vgl. Hoefele \& Konstantinidou, 2016: 151). Zur Beantwortung dieser Frage ist nicht nur eine systematische Erfassung der demographischen, migrations- und sprachbiographischen Merkmale, sondern auch eine differenzierte 
Analyse ihrer sprachlichen Fähigkeiten nötig. Im Folgenden werden zunächst die Migrations- und Sprachbiographien von Lernenden in der beruflichen Bildung und ihre Konsequenzen für die Förderung der Schreibkompetenzen theoretisch reflektiert (Kap. 2), um dann zu zeigen, wie Korpora von Lernenden-Texten zur Analyse und bedürfnisorientierten Förderung der sprachlich-kommunikativen Kompetenzen dienen können (Kap. 3-5).

\section{Erst- und Zweitspracherwerb im Kontext der Migrations- und Sprachbiographien}

Die Migrationsforschung unterscheidet verschiedene Migrationsbiographien nach Migrationshintergrund der Eltern bzw. der weiblichen oder männlichen Bezugspersonen sowie nach Alter des Kindes / der Kinder zum Zeitpunkt der Migration (vgl. Rumbaut, 2004; Portes \& Rumbaut, 2001). Von Migrationshintergrund wird gesprochen, wenn mindestens ein Elternteil im Ausland geboren ist (vgl. Brinkmann Marschke, 2014: 12 ff.; Settelmayer \& Erbe, 2010: 16; Rumbaut, 2004: 1168). Im Ausland geborene Eltern gelten als erste (Migrations-)Generation. Die zweite Generation, also Kinder, die im Aufnahmeland geboren sind oder in der frühen Kindheit migriert sind, weisen bezüglich der Sprachbiographien unterschiedliche Voraussetzungen auf, je nachdem, ob beide Eltern die gleiche Herkunftssprache oder unterschiedliche sprechen; ob ein Elternteil oder beide die Sprache des Aufnahmelandes kaum oder fliessend sprechen; welche Sprache/n in der Familie gesprochen werden; ob sie plurilingual aufwachsen oder sukzessiv mehrsprachig; wann sie in Kontakt mit der Zweitsprache gekommen sind; wie gewandt die Eltern in der Herkunftssprache, ggf. auch in der Umgebungssprache sind usw. Für Letzteres gilt der sozioökonomische Index (SEI) der Familie als zuverlässiger Indikator (vgl. Entorf, 2005: 142 ff.; Marschke, 2014: 71 ff.).

In Hinblick auf die Lese- und Schreibkompetenzen sei hier auch auf die Diglossiesituation der deutschsprachigen Schweiz verwiesen (vgl. Ammon et al., 2004; Hägi \& Scharloth, 2005). Hier findet der ungesteuerte Erst- und Zweitspracherwerb wie etwa in der Schweiz - zunächst im umgangssprachlich-dialektalen Umfeld statt, während der Erwerb der Lese- und Schreibkompetenz im schulischen Kontext im (schweizer)deutschen Standard erfolgt, was eine zusätzliche Schwierigkeit im Erwerb der Schriftsprachlichkeit darstellt, auf die der Beitrag fokussiert.

Daten hinsichtlich der Migrations- und Sprachbiographien der Lernenden (Migrationshintergrund, Migrationsalter, Erst- und Zweitsprache, Fremdsprachen, am häufigsten gesprochene Sprache, Familiensprache, Dialektgebrauch usw.) sowie Einstellungen der Auszubildenden gegenüber Erst- bzw. Zweitsprache, 
Dialekt und Standard, die einen Einfluss auf die sprachlich-kommunikativen Kompetenzen der Lernenden haben können, werden via Fragebogen erhoben. Die Variablen zu den migrations- und sprachbiographischen Merkmalen der Lernenden werden den Texten im Korpus zugeordnet (Metadaten) und diese in Hinblick auf die Textqualität analysiert.

\section{Korpusanalysen zur Förderung von Schreibkompetenzen in Berufsfachschulen}

Zur wirksamen Förderung der Schreibkompetenz bedarf es einer differenzierten Erhebung der Qualität von Lernenden-Texten. Als forschungsmethodischer Zugang hat sich bewährt, von einem möglichst großen Korpus von Lernenden-Texten unterschiedlicher Textsorten auszugehen, um sie mit Hilfe quantitativer korpuslinguistischer Methoden auf ihre sprachlich-textuellen Merkmale hin zu untersuchen (vgl. Lüdeling et al., 2005; Gries \& Stefanowitsch, 2006). Eine korpusbasierte, quantitative Analyse der sprachlichen Merkmale von Lernenden-Texten - nach Textlänge, Satzkomplexität, Wortschatzumfang, Textprozeduren resp. Prozedurausdrücken zur Bezeichnung von Textstrukturen bzw. -mustern, Register usw. (vgl. Grabowski et al., 2014; Becker-Mrotzek \& Böttcher, 2012) - kann daher dazu dienen, differenzierte schreibdidaktische Schlussfolgerungen zu ziehen, die den heterogenen Fähigkeiten und unterschiedlichen Sprachbiographien der Schüler/innen in der Berufsbildung gerecht werden (vgl. Hoefele \& Konstantinidou, 2016: 151).

\subsection{Grundgrößen der Textqualität}

\subsubsection{Wort-, Satz- und Textlänge}

Textqualität ist rein quantitativ an sich nur schwer zu erfassen; dennoch gibt es statistische Maße wie die Textlänge, die recht hoch mit der Textqualität korreliert; sie ist in methodischer Hinsicht ein höchst objektives und reliables Maß. Längere Texte sind hinsichtlich der Textqualität in der Regel die besseren, und dies über verschiedene Textsorten wie Bericht, Instruktion, Argumentation hinweg (vgl. Grabowski et al., 2014: 152). Neben der Textlänge gehören auch die Wort- und Satzlänge $z u$ den Grundgrößen, die ihr diagnostisches Potenzial in vielen automatischen Verfahren erwiesen haben (vgl. Lu, 2012; Ai \& Lu, 2013). Diese quantitativen Merkmale können automatisch erfasst und deren Zusammenhang mit der funktionalen Qualität der Lernenden-Texte geprüft werden. 


\subsubsection{Wortschatzdichte und -variation}

Ein weiteres statistisches Maß der Textqualität ist die Typetoken-Ratio, eine der gebräuchlichsten Methoden zur Bestimmung der lexikalischen Dichte und Variation. Die Wortschatzdichte wird ,weitgehend bestimmt durch das Verhältnis von bedeutungstragenden und nicht-bedeutungstragenden grammatischen Wörtern“ (Crawford \& Camiciottoli, 2007: 73). Andere Studien erfassen den Wortschatz nach dem Grad der Gebräuchlichkeit von Wörtern.

Unter Berücksichtigung der Tatsache, dass die Wortschatzdichte und -variation ein zuverlässiger Prädiktor der Textqualität ist (vgl. Nizonkiza, 2011: 122; Grabowski et al., 2014: 152), kann dieses Maß nützliche Informationen über die Wortschatzressourcen der Lernenden und Hinweise zur bedürfnisorientierten Förderung der allgemeinen Schreibkompetenz liefern.

\subsubsection{Syntaktische Muster}

Syntaktische Muster bilden in Anlehnung an das Zürcher Textanalyseraster (Nussbaumer, 1996) eine weitere Größe, die Hinweise auf die Qualität von Texten geben kann (vgl. Becker-Mrotzek \& Böttcher, 2012: 126 ff.). So hat sich gezeigt, dass beispielsweise die Art und die Anzahl der Nominalphrasen und/ oder die Komplexität der Verbalphrasen (z. B. die Anzahl der Wörter, die dem Hauptverb vorausgehen) eine Aussage über die Einfachheit bzw. Komplexität des Satzbaus ermöglichen (McNamara et al., 2011: 3). Darüber hinaus zeigt sich die Einfachheit bzw. Komplexität des Satzbaus an der Zahl der verschiedenen Satzebenen. Die funktionale Angemessenheit der syntaktischen Muster erweist sich jedoch nur in Hinblick auf Inhalt, Textsorte und Adressat und kann so gesehen - als wesentliches Merkmal der Textqualität verschiedener Textsorten und Adressaten gelten (vgl. Nussbaumer, 1996; Becker-Mrotzek \& Böttcher 2012: 131).

\subsection{Phrasen}

\subsubsection{Chunks}

Chunks als wiederkehrende Wortreihen sind textsorten- und situationsspezifischer als die einzelnen Wörter, aus denen sie gebildet werden (vgl. Barkowski \& Krumm, 2010: 34). Die Vermittlung von Chunks erhält deshalb große Bedeutung bei der Förderung von Schreibkompetenzen vor allem von „basic 
writers“ (Saughnessy, 1977). Das Erlernen von Chunks erfolgt ähnlich wie das Wortschatzlernen und setzt wenig Grammatikkenntnisse oder metalinguistisches Wissen voraus. Als kognitiv abgespeicherte Einheiten dienen sie der Entwicklung von Schreibroutinen und der Schreibflüssigkeit (vgl. Lenz et al., 2009: 48).

Die Unterstützung („Scaffolding“, vgl. Gibbons, 2002) des Schreibens durch Formulierungshilfen im Sinne von Chunks erfolgt bereits umfassend sowohl in Primar- und Sekundarschule (vgl. Bachmann \& Feilke, 2014; Philipp, 2012) wie auch in der Erwachsenenbildung in DaF/DaZ-Kursen (Lewis, 1993; Lenz et al., 2009: 48). Unter den Konzepten, die den Formulierungsprozess beim Schreiben unterstützen, versprechen korpusbasierte Ansätze, die Chunks erfassen und bereitstellen, vermutlich den größten Nutzen. Ihre besondere Stärke hängt mit der Möglichkeit zusammen, dass die sprachlichen Ressourcen, u. a. auch Chunks, differenziert nach Nutzergruppen, Textsorten, Allgemein- oder Fachsprachen identifiziert und gefördert werden können. Korpora ermöglichen zudem, die halbautomatische Extraktion von für die Routine von Formulierungsprozessen essenziellen sprachlichen Konstrukten ganz zu automatisieren.

\subsubsection{Textprozeduren}

Textprozeduren sind relevant als Beschreibungsmerkmal von textsortenspezifischen Mustern, aus didaktischer Sicht daher für den Erwerb von Schreibkompetenzen. Eine Textprozedur ist eine „Einheit von Handlungsschema und Ausdruck“ (Feilke, 2014: 14); es ist ein textuelles, semiotisches Zeichen, das aus einer Sprachhandlung entsteht und ein Textmuster bezeichnet (z. B. zeigen, dass etwas zu einem Ende kommt durch „Abschließend lässt sich sagen“). Textprozeduren stellen gemäß Feilke (2014: 14) eine mittlere Einheit zwischen Satz und Text dar.

Laut Feilke und Bachmann (2014: 8) sind Textprozeduren „die grundlegende didaktische Bezugsgröße für eine Schreibdidaktik, die das sprachliche Textwissen einbeziehen und für die Förderung der Schreibkompetenz nutzen möchte“, da Textprozeduren „sozial schematisierte Handlungszüge in Texten“ und „Werkzeuge des Schreibens“ darstellen. Prozedurausdrücke können als Hinweise auf die Strukturiertheit von Texten betrachtet werden und somit auch als Indikatoren für Textqualität gelten (vgl. Knopp et al., 2014). 


\subsection{Sprachebene}

\subsubsection{Allgemeine Parameter der Sprachebene}

Neben der eher traditionellen Perspektive der Betrachtung anhand von Merkmalen des mündlichen oder schriftlichen Registers haben Forschende die Menge an detaillierten linguistischen Eigenschaften betrachtet, welche die Fluktuation im Register markieren. So identifizierte zum Beispiel Giménez-Moreno (2011: 19) zehn linguistische Parameter von Sprachebenen in der beruflichen Kommunikation (s. Tab. 1). Diese Parameter haben ein erhebliches Potenzial, relevante linguistische Textsorten-Deskriptoren im Kontext der beruflichen Bildung zu sein.

Tab. 1: Parameter der Sprachebenen in der beruflichen Kommunikation (Giménez-Moreno, 2011: 19)

A B

\section{(+) Informal/zufällig}

Persönliche Ausdrucksformen

Aktive Verben/Redewendungen

Direkte Rede

Gewöhnliche berichtende Verben

(z. B. sagen)

Gewöhnliche Konnektoren

(z. B. so)

Allgemeinbegriff/Ausdrucksformen

(z. B. Mann)

Emotionale/subjektive/

einstellungsbezogene Begriffe

(z. B. vermuten)

Informelle Redewendungen

Nutzung von Schmelzwörtern, Abkürzungen

und schnelle Ausdruckweise

Klare Aussagen und direkte Anweisungen

\section{(+) Formal/rituell}

Unpersönliche Ausdrucksformen

Passive Verben/Redewendungen

Indirekte Rede

Spezifische berichtende Verben

(z.B. nennen)

Eher umfangreiche Konnektoren

(z. B. darüber hinaus)

Genaue Bestimmungen/Ausdrucksformen

(z. B. Techniker)

Neutrale/objektive Begriffe

(z. B. informieren)

Hochsprachliche formale Ausdrücke

Detaillierte und konkrete Ausdrucksformen ohne Kürzel unter Einsatz von Nominalisierungen und Bestimmungswörtern Kennzeichnungen von Höflichkeit, Zurückhaltung und Milderung

\subsubsection{Parlando}

Berufsfachschüler/innen weisen nicht selten Probleme im Gebrauch der formalen Sprache auf. Sie neigen dazu, in ihren Texten informale Sprache zu verwenden, und schreiben oft, wie sie sprechen (Parlando) (vgl. Sieber, 1998; Efing, 2008). 
Rechts- und Fachtexte zum Beispiel, die in der beruflichen Bildung eine gewisse Rolle spielen, sind durch einen relativ hohen Grad an Nominalisierungen charakterisiert, die deren Komplexität und Dichte erhöhen. Insofern stellen sie eine Herausforderung vor allem für jene Berufsfachschüler/innen dar, die einen begrenzten Kontakt mit Bildungssprache bzw. formaler Sprache haben (vgl. Jahr, 1996: 12). In Komplexitäts-Messstudien definieren sich die Merkmale der mündlichen, informalen Sprache durch die Nähe oder Distanz zu linguistischen Parametern von Formalität (vgl. Graesser et al., 2014: 212). Formale Texte sind gekennzeichnet durch linguistische Merkmale wie „abstrakte Wörter, komplexe Syntax und Kohäsion“ (Graesser et al., 2014: 219), die in die Analyse einzubeziehen sind.

\subsection{Textsortenspezifika}

Für eine genauere Messung der Textqualität empfiehlt es sich, die dargestellten Merkmale anhand verschiedener Textsorten (narrativ, informativ, persuasiv) zu erheben (vgl. Grabowski et al., 2014). Der Begriff Textsorte verweist auf Regelhaftigkeiten des Sprachgebrauchs und damit auf charakteristische Merkmale bestimmter Texte (vgl. Hyland, 2000). Diese Regelhaftigkeiten sind nach Hyland nicht einfach stilistische Varianten, sondern Abbildungen unterschiedlicher sozialer Handlungen, die mittels Sprache vollzogen werden und sich durch unterschiedliche pragmatische Erwartungen in Bezug auf die Textsorte und die Textwirkung konstituierten. Im engeren Sinn können unter „Textsorte“ Textmuster verstanden werden, die aus der wiederkehrenden Verwendung bestimmter Ausdrücke mit einem definierten Zweck in gewissen sozialen Kontexten hervorgehen (vgl. Bazerman, 1994; Russell, 1997). - Die textsortenbasierte Korpuslinguistik (vgl. Nesi \& Gardner, 2012; Swales, 2004) hat Erkenntnisse bezüglich allgemeiner, den sprachlichen Ausdruck betreffender Profile sowie hinsichtlich textsortenspezifischer Strukturen und Funktionen geliefert, die hier zur Verwendung kommen.

\section{Korpusanalysen und Textqualität}

Zur Bewertung der Textqualität kann man sich - zusätzlich zur Korpusanalyse auf das für den Kontext der Berufsbildung und im Rahmen des Projekts PROSAB (vgl. Konstantinidou et. al., 2016) entwickelte Kodierbuch stützen, das von einer Mischmethode von analytischem und holistischem Scoring ausgeht. Das Kodierbuch für die Bewertung der Textqualität von Berufsfachschülern/-schülerinnen 
mit drei Basisdimensionen und je zwei Subskalen (Kriterienkomplexen) wurde in Anlehnung an die Beurteilungsverfahren von DESI, VERA-8, das Zürcher Textanalyseraster und den Katalog von Becker-Mrotzek \& Böttcher (2012) entwickelt (vgl. Konstantinidou et. al., 2016: 83 ff.). Die Beschränkung auf nur sechs Subskalen, die jeweils mehrere Kriterien umfassen, liegt in der größeren Praktikabilität des Beurteilungsverfahrens begründet. So empfehlen Baurmann (2008: 133) sowie Becker-Mrotzek \& Böttcher (2012: 128) eine mittlere Anzahl von Einzelkriterien, die an verschiedene Textsorten - narrative, informative und persuasive - anpassbar sind. Die drei Basisdimensionen mit je zwei Subskalen stellen sich so dar:

1. Sprachliche Kompetenzen (sprachliche Richtigkeit, Wortwahl)

2. Textsortenbezogene Kompetenzen (formale Textsortenkonventionen Struktur / roter Faden)

3. Pragmatische Kompetenzen (Inhalt und kommunikative Wirkung)

Die Gesamtbewertung der Textqualität ergibt sich aus den sechs Subskalen, die jeweils fünf Abstufungen aufweisen (4) und alle gleich gewichtet sind (vgl. Konstantinidou et. al., 2016: 83 ff.).

Da durch das Korpus eine detaillierte linguistische Analyse der Texte und das Erfassen gewisser Merkmale der Textqualität gewährleistet sind, kann man durch holistisches Rating die Dimensionen erfassen, die mit dem Korpus schwer zu erfassen sind. Durch Rater zu bewerten ist nämlich vor allem, ob der Text inhaltlich so bearbeitet wurde, dass er seine kommunikative Funktion in Hinblick auf den Adressaten erfüllt (pragmatische Kompetenzen). Ein weiterer Aspekt, der aufgrund seiner Wichtigkeit für die Bestimmung der Qualität der Texte (vgl. Konstantinidou et al., 2016: $91 \mathrm{f}$.) interessiert und allein durch das Korpus nur schwer zu erfassen ist, ist die Textstruktur: „Ist der Text sinnvoll und logisch aufgebaut?“ Schließlich sollte die sprachliche Richtigkeit nicht analytisch im Sinne der Regelverstöße, sondern im Sinne der Textwirkung ebenfalls durch Rating erfasst werden: „Treten im Text Fehler in der Orthografie, im Satzbau und/oder in der Grammatik auf, die den Lesefluss oder die Verständlichkeit beeinträchtigen?“

Auf der Grundlage der beschriebenen Verfahren zur Erhebung der Textqualität werden Texte mit ähnlichen bzw. typischen Merkmalen extrahiert und so Cluster von Textmerkmalen in Verbindung mit migrations- und sprachbezogenen Variablen der Lernenden gebildet. Die Clusterbildung erlaubt eine für die Berufsbildung bis anhin nicht existierende quantitative, korpusbasierte Beschreibung der sprachlich-kommunikativen Kompetenzen nach Lernenden-Profilen. Ihr kommt ein hohes diagnostisches Potenzial zur differenzierten Bestimmung des Förderbedarfs im Bereich (schrift)sprachlich-kommunikativer Kompetenzen zu, und zwar der Lernenden insgesamt wie auch einzelner Lernendengruppen bzw. Klassen. 


\section{Nutzen der Ergebnisse für die Praxis der Förderung von sprachlich-kommunikativen Kompetenzen}

Der Nutzen für die Praxis der Förderung von sprachlich-kommunikativen Kompetenzen im Kontext der beruflichen Bildung besteht darin, dass mit dem Korpus bzw. den Clustern ein hocheffizientes (elektronisches) Werkzeug existiert, das Schwächen und Stärken von Lernenden-Texten erkennt und wertvolle Informationen zur Lernzielbestimmung und zur evidenzbasierten individuellen Förderung der Schreibkompetenz liefert.

Darauf aufbauend können - unter Rückgriff auf die Ergebnisse der bereits abgeschlossenen Studie zur prozessorientierten Schreibdidaktik zwischen Deutsch als Mutter-(DaM) und Zweitsprache (DaZ) (vgl. Hoefele \& Konstantinidou, 2016: 151) - differenzierte mutter- bzw. zweitsprachendidaktische Ansätze zur Förderung der allgemeinen Schreibkompetenz systematisch dargestellt werden, die individuell auf die erhobenen Cluster bzw. die Lernenden-Profile abgestimmt sind. Die Ergebnisse stellen eine empirisch gesicherte Grundlage zur Förderung der Grundkompetenzen dar, insbesondere des Schreibens in der Berufsbildung, die der sprachlichen Heterogenität der Lernenden Rechnung trägt; sie können wesentliche Impulse für die Curriculum-Entwicklung wie auch für die Aus- und Weiterbildung der Lehrpersonen des allgemeinbildenden Unterrichts (ABU) an Berufsfachschulen geben.

Das aufgebaute Korpus legt darüber hinaus den Grundstock für die Weiterentwicklung der Berufsbildungsforschung im Bereich der Sprachförderung, so z. B. für bisher fehlende Längsschnittstudien, die Aussagen über die Entwicklung der sprachlich-kommunikativen Kompetenzen der Lernenden über die Zeit ermöglichen.

\section{Literaturverzeichnis}

Ai, H., \& Lu, X. (2013). A corpus-based comparison of syntactic complexity in NNS and NS university students writing. In Ballier, N., Díaz-Negrillo, A. \& Thompson, P. (eds.). Automatic treatment and analysis of learner corpus data (pp. 249-264). Amsterdam: John Benjamins.

Ammon, U., Bickel, H., \& Ebner, J. (2004). Variantenwörterbuch des Deutschen: Die Standardsprache in Österreich, der Schweiz und Deutschland sowie in Liechtenstein, Luxemburg, Ostbelgien und Südtirol. Berlin: Walter de Gruyter.

Bachmann, T., \& Feilke, H. (Hrsg.). (2014). Werkzeuge des Schreibens. Beiträge zu einer Didaktik der Textprozeduren. Stuttgart: Fillibach bei Klett. 
Barkowski, H., \& Krumm, H.-J. (2010). Fachlexikon Deutsch als Fremd- und Zweitsprache. Tübingen: Narr Francke Attempto Verlag.

Baurmann, J. (2008). Schreiben. Überarbeiten. Beurteilen. Ein Arbeitsbuch zur Schreibdidaktik (3. Aufl.). Seelze-Velber: Kallmeyer.

Baumann, K. (2014). „Man muss schon ein bisschen mit dem Schreiben zurechtkommen!“ Eine Studie zu den Schreibfähigkeiten von Auszubildenden im unteren beruflichen Ausbildungssegment im Kontext von Ausbildungsreife. Paderborn: Eusl Verlagsgesellschaft.

Bazerman, C. (1994). Systems of genres and the enactment of social intentions. In Freedman, A. \& Medway, P. (eds.). Genre and the new rhetoric (pp. 79-101). London: Taylor \& Francis.

Becker-Mrotzek, M. \& Böttcher, I. (2012). Schreibkompetenz entwickeln und beurteilen. (4. Aufl.). Berlin: Cornelsen Scriptor.

Crawford Camiciottoli, B. (2007). The language of business studies lectures: A corpus-assisted analysis. (Pragmatics \& Beyond New Series 157). Amsterdam: Benjamins.

Economiesuisse (2010). Volksschule: Fokus auf das Wesentliche. Economiesuisse: Zürich.

Efing, C. (2008). „Aber was halt schon schwer war, war, wo wir es selber schreiben sollten.“ Defizite und Förderbedarf in der Schreibkompetenz hessischer Berufsschüler. In Jakobs, E.-M. \& Lehnen, K. (Hrsg.). Berufliches Schreiben. Ausbildung, Training, Coaching (S. 17-34). Frankfurt/M.: Peter Lang.

Entorf, H. (2005). PISA-Ergebnisse, sozioökonomischer Status der Eltern und Sprache im Elternhaus. Eine international vergleichende Studie vor dem Hintergrund unterschiedlicher Einwanderungsgesetze. Tertium comparationis 11(2), 134-151.

Feilke, H. (2014). Argumente für eine Didaktik der Textprozeduren. In Bachmann, T. \& Feilke, H. (Hrsg.). Werkzeuge des Schreibens. Beiträge zu einer Didaktik der Textprozeduren (S. 11-34). Stuttgart: Fillibach bei Klett.

Gibbons, P. (2002). Scaffolding Language. Scaffolding Learning. Teaching Second Language Learners in the Mainstream Classroom. Portsmouth (N.H.): Heinemann.

Giménez-Moreno, R. (2011). Register variation in international business correspondence. International Journal of English Studies 11(1), 15-34.

Grabowski, J., Becker-Mrotzek, M., Knopp, M., Jost, J. \& Weinzierl, Chr. (2014). Comparing and combining different approaches to the assessment of text quality. In Knorr, D. Heine, C. \& Engberg, J. (eds.). Methods in writing process research (pp. 147-165). Frankfurt/M.: Peter Lang.

Graesser, A. C., McNamara, D. S., Cai, Z., Conley, M., Li, H., \& Pennebaker, J. (2014). Coh-Metrix measures text characteristics at multiple levels of language and discourse. The Elementary School Journal 115(2), 210-229.

Gries, S., \& Stefanowitsch, A. (2006). Corpora in cognitive linguistics: corpus-based approaches to syntax and lexis. Berlin: Mouton de Gruyter.

Hägi, S. \& Scharloth, J. (2005). „Ist Standarddeutsch für Deutschschweizer eine Fremdsprache? Untersuchungen zu einem Topos des sprachreflexiven Diskurses“. Linguistik online 24(3), 19-47.

Hoefele, J. \& Konstantinidou, L. (2016). Förderung der allgemeinen Schreibkompetenz an Berufsschulen: Prozessorientierte Schreibdidaktik zwischen DaM (Deutsch als Muttersprache) und DaZ (Deutsch als Zweitsprache). In Kreyer, R., Güldenring, B. \& Schaub, S. (Hrsg.). Angewandte Linguistik in Schule und Hochschule. Neue Wege für Sprachunterricht und Ausbildung (S. 135-163). Frankfurt/M.: Peter Lang.

Hoefele, J., Konstantinidou, L. \& Weber C. (2017). Zweitsprachendidaktische Konzepte in der Förderung der allgemeinen Schreibkompetenz an Berufsfachschulen. In Efing, C. \& Kiefer, 
K.-H. (Hrsg.). Sprachbezogene Curricula und Aufgaben in der beruflichen Bildung. Bern: Peter Lang.

Hyland, K. (2000). Disciplinary Discourses: Social Interactions in Academic Writing. London: Longman.

Jahr, S. (1996). Das Verstehen von Fachtexten. Tübingen: Narr.

Knopp, M., Jost, J. Linnemann, M. \& Becker-Mrotzek, M. (2014). Textprozeduren als Indikatoren von Schreibkompetenz - ein empirischer Zugriff. In Bachmann, T. \& Feilke, H. (Hrsg.). Werkzeuge des Schreibens. Beiträge zu einer Didaktik der Textprozeduren (S. 111-128). Stuttgart: Fillibach bei Klett.

Konstantinidou, L., Hoefele, J., \& Kruse, O. (2016). Assessing writing in vocational education and training schools: Results from an intervention study. In Göpferich, S. \& Neumann, I. (eds.). Assessing and developing writing skills (pp. 73-102). Tübingen: Narr.

Lenz, P., Andrey, S., \& Lindt-Bangerter, B. (2009). Rahmencurriculum für die sprachliche Förderung von Migrantinnen und Migranten. Bern: Bundesamt für Migration.

Lewis, M. (1993). The Lexical Approach. Hove: Language Teaching Publications.

$\mathrm{Lu}, \mathrm{X}$. (2012). The relationship of lexical richness to the quality of ESL learners' oral narratives. The Modern Language Journal 96(2), 190-208.

Lüdeling, A., Walter, M., Kroymann, E., \& Adolphs, P. (2005). Multi-level error annotation in learner corpora. Proceedings of Corpus Linguistics, Birmingham.

Marschke, B. (2014). Gesellschaftliche Teilhabe und Chancengleichheit als Indikatoren für Integration. In Marschke, B. \& Brinkmann, H.U. (Hrsg.). Handbuch Migrationsarbeit (2. Aufl.) (S. 61-78). Wiesbaden: Springer VS.

McNamara, D. S., Graesser, A. C., Cai, Z., \& Kulikowich, J. M. (2011). Coh-metrix easability components: aligning text difficulty with theories of text comprehension. AERA.

Nesi, H., \& Gardner, S. (2012). Genres across the disciplines: student writing in higher education. Cambridge: Cambridge University Press.

Nizonkiza, D. (2011). The relationship between lexical competence collocational competence and second language proficiency. English Text Construction 4(1), 113-145.

Nussbaumer, M. (1996). Lernerorientierte Textanalyse - Eine Hilfe zum Textverfassen? In Feilke, H. \& Portmann P.R. (Hrsg.). Schreiben im Umbruch. Schreibforschung und schulisches Schreiben (S. 96-112). Stuttgart etc.: Ernst Klett Verlag.

Philipp, M. (2012). Besser lesen und schreiben. Wie Schüler effektiver mit Sachtexten umgehen lernen. Stuttgart: Kohlhammer.

Portes, A. \& Rumbaut, R. G. (2001). Legacies: The Story of the Immigrant Second Generation. Berkeley, New York: University of California Press and Russell Sage Foundation.

Rumbaut, R. G. (2004). Ages, Life Stages, and Generational Cohorts: Decomposing the Immigrant First and Second Generations in the United States. International Migration Review, 38 (3), 1160-1205.

Russell, D. (1997). Rethinking Genre in School and Society: An Activity Theory Analysis. Written Communication 14(4), 504-554. Retrieved 22.02.2017 from http://www.public.iastate. edu/ drrussel/at\&genre/at\&genre.html

Saughnessy, M. (1977). Errors and Expectations. New York: Oxford University Press. 
Sieber, P. (1998). Parlando in den Texten zur Veränderung kommunikativer Grundmuster in der Schriftlichkeit. Tübingen: Niemeyer.

Swales, J.M. (2004). Research genres. Explorations and applications. Cambridge: Cambridge University Press. 